\title{
INTOXICAÇĀO ALIMENTAR POR QUEIJO MINAS CONTAMINADO COM STAPHYLOCOCCUS AUREUS
}

\author{
José Geraldo Sabioni* \\ Elisa Yoko Hirooka** \\ Maria de Lourdes R. de Souza**
}

\begin{abstract}
SABIONI, J.G. et al. Intoxicação alimentar por queijo Minas contaminado com Staphylococcus aureus. Rev. Saúde públ., S. Paulo, 22:458-61, 1988.

RESUMO: Relata-se surto de intoxicação alimentar ocorrido em julho de 1987, na cidade de Ouro Preto, MG (Brasil). O alimento causador foi um queijo Minas, contaminado por Staphylococcus aureus ao nivel de $9,3 \times 10^{7} \mathrm{UFG} / \mathrm{g}$. Detectaram-se cepas produtoras de enterotoxinas do tipo A,B,D e E. A amostra analisada revelou contaminação por coliformes fecais acima de $1,1 \times 10^{5} / \mathrm{g}(\mathrm{NMP})$, mas não continha Salmonella.Devido aos sintomas característicos e à elevada contaminação, concluiu-se que o Staphylococcus aureus foi o patogênico responsável pelo surto.
\end{abstract}

UNITERMOS: Intoxicação alimentar estafilocócica, incidência. Staphylococcus aureus, análise. Queijo, efeitos adversos. Surtos de doenças, incidência.

\section{INTRODUÇÃO}

O queijo "Minas", produto amplamente consumido no Brasil, é na maioria das vezes fabricado com leite cru, em fazendas. Deste modo, a possibilidade de persistência do S.aureus, no queijo, é grande, com conseqüente produção de toxinas ${ }^{13,14,19}$. Em Minas Gerais, Estado tradicionalmente produtor de queijo, esta situação agrava-se ainda mais, pois, de acordo com os estudos de Ferreira, em 1979, citado por San$\operatorname{tos}^{13}$, cerca de $40 \%$ dos rebanhos leiteiros do Sudeste de Minas apresentaram a mamite. Além deste ponto de contaminação, somam-se a transmissão do patogênico ao leite pelo homem durante e após a ordenha, as precárias condições higiênicas na fabricação do queijo e a permanência do produto, desde a produção até sua comercialização, em temperaturas que suportam o crescimento do Staphylococcus aureus, bem como de outros patogênicos ${ }^{2,3,11,14}$. Devido a esses fatores, a maioria dos queijos "Minas" comercializados no Brasil não apresentam qualidade microbiológica satisfatória, principalmente em relação ao patogênico S.aureus. Os riscos de intoxicação são constantes conforme mostraram os estudos já realizados s $^{3,8,10}$.

No presente trabalho os autores relatam um surto de intoxicação alimentar, ocorrido em uma família de baixa renda, que ingeriu queijo contaminado com S.aureus enterotoxigênico.

\section{MATERIAL E MÉTODOS}

Em 20 de julho de 1987, o proprietário de uma mercearia de Ouro Preto (MG) solicitou ao Laboratório de Higiene de Alimentos do Departamento de Nutrição da Escola de Farmácia da Universidade Federal de Ouro Preto uma análise microbiologica de uma amostra de queijo "Minas"-frescal, suspeito de ter causado uma intoxicação alimentar em uma família composta de quatro membros (pai, mãe, filho, filha), que ingeriu o queijo durante um lanche no período da tarde.

Foi efetuada contagem de Staphylococcus aureus utilizando-se "Agar Baird Parker", coliformes fecais através do Número Mais Provável em caldo E.C., e a pesquisa de Salmonello em $25 \mathrm{~g}$ do produto, conforme recomendado pela ICMSFs.

Dez colônias foram isoladas a partir do crescimento em "Agar Baird Parker" e submetidas aos testes bioquímicos da coagulase e termonuclease para identificação do S.aureus ${ }^{5}$. Após identificação, as culturas foram analisadas quanto à enterotoxigenicidade, no Departamento de Patologia Geral do Centro de Ciên-

\footnotetext{
* Departamento de Nutrição da Escola de Farmácia/da Universidade Federal de Ouro Preto "Campus" - Morro do Cruzeiro - 35400 - Ouro Preto, MG - Brasil.

** Departamento de Patologia Geral do Centro de Ciências Biológicas da Fundação Universidade Estadual de Londrina - Caixa Postal 6001 - 86051 - Londrina, PR - Brasil.
} 
cias Biológicas da Universidade Estadual de Londrina. Para a deteç̧ão da enterotoxina, cada cultura foi crescida em caldo BHI (Biobrás), durante uma noite, a $37^{\circ} \mathrm{C}$. Em seguida, $0,1 \mathrm{ml}$ da cultura era transferido para placa contendo caldo BHI, $1 \%$ de extrato de levedura e celofane ("Arthur H. Thomas" Scientific Products) e incubado por $24 \mathrm{~h} / 37^{\circ} \mathrm{C}$. Após incubação eram adicionados $1,5 \mathrm{ml}$ de $\mathrm{Na}_{2} \mathrm{HPO}_{4} 0,01 \mathrm{M}$ e centrifugado a $15.000 \mathrm{rpm}$ em microcentrífuga. $O$ sobrenadante foi utilizado para detecção da enterotoxina pelo método OSP (Optimum Sensitivity Plate) $)^{5}$ O ágar para imunodifusão era constituido de tampão TRIS 0,05M, pH 7,4, $\mathrm{NaCl} 0,85 \%$, mertiolate $1: 10.000$ e $1,2 \%$ de ágar de alta pureza.

Os sintomas e o período de incubação do surto, bem como informações adicionais, foram levantados através de entrevista com os membros da família que sofreram a intoxicação alimentar.

\section{RESULTADOS E DISCUSSÃO}

Os resultados da análise microbiológica da amostra de queijo estão representados na Tabela 1. Nota-se que a amostra de queijo não atendeu aos padrơes vigentes no Brasil $^{2}$, com relação ao microrganismo S.aureus e coliformes fecais, o que é comum nos queijos "Minas", conforme resultados obtidos por outros pesquisadores ${ }^{3,8,10}$. O nível de contaminação encontrado para o S.aureus mostra que a amostra de queijo analisada já se classifica como alimento infeccioso, pois é superior a $10^{6} \mathrm{UFC} / \mathrm{g}^{6}$. Mesmo com elevada contaminação por coliformes fecais, não foi detectada a presença de Salmonella em $25 \mathrm{~g}$ do produto.

$\mathrm{Em}$ face do resultado anterior, foram levantados, por meio de inquérito, os sintomas, período de incubação do surto e quais outros tipos de alimentos a família havia consumido durante aquele dia.

Os sintomas predominantes ocorridos em ordem foram náuseas, vômitos, diarréia, dores abdominais. A não observância da febre dentre os sintomas indicam um caso típico de intoxica-

\section{TABELA 1}

Resultados da análise microbiológica da amostra de queijo, em surto de intoxicação. Ouro Preto (MG), 1987.

\begin{tabular}{lcc} 
Microrganismos & \multicolumn{2}{c}{ Nível de Contaminação } \\
\hline Staphylococcus aureus & UFC/g & $9,3 \times 10^{7}$ \\
Coliformes fecais & NMP $/ 8$ & $1,1 \times 10^{5}$ \\
Salmonella & em $25 \mathrm{~g}$ & negativo \\
\hline
\end{tabular}

ção alimentar. O período de incubação foi em média de uma hora.

Uma vez que o número de casos foi apenas quatro, não foi construída a tabela do Índice de Ataque. De acordo com o inquérito, toda a família tinha almoçado naquele dia, arroz, feijão, couve e angú, preparados no mesmo dia, por volta das $11 \mathrm{~h}$. No período da tarde foi servido um lanche composto de café, queijo e pão. $\mathrm{Pai}, \mathrm{mãe}$ e filha ingeriram o lanche e após uma hora apresentaram sintomas de intoxicação, tendo mãe e filha sido internadas. O filho, como foi para casa de parentes na parte da tarde, não ingeriu o lanche e não foi contaminado naquele dia. Ao saber do caso, no dia seguinte, retornou para casa, comeu o queijo e foi intoxicado. Essas observações oferecem fortes evidências de que o queijo foi o alimento causador da intoxicação.

Devido à elevada contaminação da amostra de queijo com S.aureus, foram isoladas dez linhagens para avaliação da existência de enterotoxigênicos. Pela Tabela 2, nota-se que $80 \%$ das culturas produziram a SEA, $20 \%$ a SEB, $30 \%$ a SED e $10 \%$ a SEE. As enterotoxinas SEC e TSST-1 não foram produzidas pelas linhagens testadas. Verificou-se que apenas as culturas 3 e 7 não produziram qualquer tipo de enterotoxina. A presença de linhagens enterotoxigênicas, juntamente com os sintomas observados no surto, sugere que S.aureus foi o responsável pela intoxicação.

Diversos trabalhos publicados por brasileiros mostraram uma constante contaminação por S.aureus no leite destinado ao fabrico do queijo, como também no produto acabado $^{3,8,10,13,14,16,19}$. Nascimento e col. ${ }^{10}$, em experimento em que se avaliou a qualidade microbiológica de queijos "Minas" frescal comercializados em Ouro Preto (MG), constataram que

\section{TABELA 2}

Estudo da presença de $S$. aureus enterotoxigênico em dez linhagens originárias de queijo "Minas", em surto de intoxicação. Ouro Preto (MG), 1987.

\begin{tabular}{ccccccc}
\hline $\begin{array}{c}\text { Linhagens } \\
N !\end{array}$ & SEA & SEB & SEC & SED & SEE & TSST-1 \\
\hline 1 & + & + & - & - & - & - \\
2 & + & - & - & + & - & - \\
3 & - & - & - & - & - & - \\
4 & + & + & - & - & - & - \\
5 & + & - & - & + & + & - \\
6 & + & - & - & - & - & - \\
7 & - & - & - & - & - & - \\
8 & + & - & - & + & - & - \\
9 & + & - & - & - & - & - \\
10 & + & - & - & - & - & - \\
\hline
\end{tabular}


$41,1 \%$ das amostras analisadas estavam contaminadas com S.aureus acima $10^{6} \mathrm{UFC} / \mathrm{g}$, portanto, potencialmente infecciosas. Mandil $e$ col. ${ }^{8}$, em experimento semelhante conduzido em Belo Horizonte, constataram que 21,5\% das amostras de queijo "Minas" apresentaram S.aureus acima de $10^{5} \mathrm{UFC} / \mathrm{g}$, sendo que $21,97 \%$ das cepas estudadas eram enterotoxigênicas.

Países desenvolvidos geralmente possuem dados epidemiológicos sobre intoxicaçðes de origem alimentar. No periodo de 1962 a 1971, cerca de $5 \%$ das intoxicaçoes alimentares relatadas na Inglaterra e Gales foram devidas ao S.aureus $^{4}$. No Canadá, durante o biênio 78.79 , ocorreram 44 surtos com 523 casos confirmados ${ }^{18}$. Nos Estados Unidos, no período de 1969 a 1973, um total de 499 surtos foram de origem estafilocócica, representado cerca de $30 \%$ dos surtos de intoxicaçঠes alimentares relatados'. Nesse mesmo país, em 1982, ocorreram 28 surtos de intoxicações estafilocócicas com 669 casos, sem registro de letalidade ${ }^{7}$.

Segundo Bergdoll ${ }^{1}$, a verdadeira incidência de intoxicação estafilocócica é desconhecida, porque a doença não é relatada na maioria dos países. No Brasil não existem dados epidemiológicos, mas é de se espear que sejam altos os indices de intoxicaçōes alimentares de origem microbiana, embora poucos sejam os casos relatados na literatura ${ }^{6,12,15,17}$.

\section{AGRADECIMENTOS}

À Farmacêutica Bioquímica do Departamento de Nutrição da Escola de Farmácia, Nair Pimenta, pela análise microbiológica realizada.

SABIONI, J.G. et al. [Food-poisoning from Minas-type cheese, contamined with Staphylococcus aureus]. Rev. Saúde públ., S. Paulo, 22:458-61, 1988.

ABSTRACT: An outbreak of food poisoning which occurred in July, 1987, in the city of Ouro Preto, State of Minas Gerais, Brazil, is reported. The food involved was a Minas-type cheese, contamined by Staphylococcus aureus to the level of $9.3 \times 10^{7} \mathrm{CFU} / \mathrm{g}$. Enterotoxin producing strains of types A,B,D and $\mathrm{E}$ were detected. The sample analysed also showed contamination by fecal coliforms above $1.1 \times 10^{5} / \mathrm{g}$ (MPN), but Salmonella were not present. Due to characteristic symptoms and high contamination it was concluded that Staphylococcus aureus was the pathogene responsible for the outbreak.

UNITERMS: Staphylococcal food poisoning, occurrence. Staphylococcus aureus, analysis. Cheese, adverse effects. Disease outbreaks, occurrence.

\section{REFERENCIAS BIBLIOGRÁFICAS}

1. BERGDOLL, M.S.S. Staphylococcal intoxication. In: Riemann, H. \& Bryan, F.L., eds. Food-borne infections and intoxications. $2^{\text {nd }}$ ed. New York, Academic Press, 1979.

2. DELAZARI, I.; LEITÃO, M.F.F.; GERALDINI, A.M.; EIROA, M.N.U.; VALLE, J.L.E. Desenvolvimento de Staphylococcus aureus e produção de enterotoxina em queijo tipo Minas. Col. Inst. Tecnol. Alim., 9:163-74, 1978.

3. FURLANETTO, S.M.P.; CERQUEIRA-CAMPOS, M.L.; IARIA, S.T. Pesquisa de Staphylococcus aureus, Bacillus cereus, Clostridium perfringens e Salmonella em queijo frescal, vendido em supermercados do município de São Paulo. In: Congresso Latino Americano de Microbiologia, 9:/ Congresso Brasileiro de Microbiologia, 12:, São Paulo, 1983. Programa-Resumos. São Paulo, 1983. p. 144.

4. GILBERT, R.J, \& WIENEKE, A.A. Staphylococcal food poisoning with special reference to the detection of enterotoxin in food. In: Hobbs, B.C. \& Christian, J.H.B., eds. The microbiological safety of food. New York, Academic Press, 1973.

5. INTERNATIONAL COMMISSION ON MICROBIOLOGICAL SPECIFICATIONS FOR FOODS. Microorganisms in foods. I - Their significance and methods of enumeration. Toronto, University of Toronto Press, 1978.
6. KARINO, E.H.; OKADA, C.; PAULA, C.H.; DOSSI, A.; MUTTA, T.; CASADO, N.Y.A.; MATOS, N.M. Relato de um surto de intoxicação alimentar por Staphylococcus aureus, em Paranavai (PR). Hig. alim., 4:102-5, 1985.

7. MAC DONALD, K.L. \& GRIFFIN, P.M. Foodborne disease outbreaks, Annual Summary, 1982. J. Food Protec., 49:933-9, 1986.

8. MANDIL, A.; MORAIS, V.A.D.; PEREIRA, M.L.; FAGUNDES, J.M.S.; CARMO, L.S.; CORREIA, M.G.; CASTRO, E.P.; GOMES, M.J.V.M. Staphylococcus aureus em queijo tipo "Minas". Cienc. Tecnol. Alim., 2:233-41, 1982.

9. MINISTÉRIO DA SAÚDE. Divisão Nacional de Vigilância Sanitária de Alimentos. Portaria No 01 de 28/01/87: Padróes microbiológicos de alimentos da DINAL. Diário Oficial da União, Brasília, $12 \mathrm{fev.}$ 1987.

10. NASCIMENTO, D.; SABIONI, J.G.; PIMENTA, N.; XANDÓ, S.R. Avaliaçåo microbiológica de queijos tipo Minas-frescal da cidade de Ouro Preto (MG). Bol.SBCTA, Campinas, 19:120-9, 1985.

11. SABIONI, J.G. \& NASCIMENTO, D. Ocorrência de Clostridium perfringens em queijos tipo Minas. frescal de Ouro Preto (MG). Hig. alim., 4:219-22, 1985.

12. SALZBERG, S.P.; MASSAGUER, P.R.; SERRANO, 
A.M. Estudo epidemiológico e microbiológico de um surto de intoxicação alimentar. Rev. Microbiol., S. Paulo, 13:26-30, 1982.

13. SANTOS, E.C. Controle da flora estafilocócica em queijo Minas durante o processamento industrial. Arq. Esc. Vet. UFMG, Belo Horizonte, 33:199-205, 1981.

14. SANTOS, E.S.; GENIGEORGES, C.; FARVER, T.B. Prevalence of Staphylococcus aureus in raw and pasteurized milk used for commercial manufacturing of Brazilian Minas cheese. J. Food Protec., 44:172-6, 1981.

15. SERRANO, A.M. Uma toxinfecção alimentar por Clostridium perfringens. Atual. vet., 28:36-9, 1976.

16. SILVA, C.A.M. Avaliação da qualidade microbiológica de queijo tipo Minas-frescal consumido na cidade do Rio de Janeiro. In: Congresso Brasileiro de Ciên- cia e Tecnologia de Alimentos, 4:, Rio de Janeiro, 1980. Programa oficial. Resumos de temas livres. Rio de Janeiro, 1980. p. 202.

17. SILVA, G.R.; SILVA, I.; GUIMARÃES, C.C. An outbreak of food poisoning due to Salmonella typhimurium in a general hospital. Rev. Inst. Med. Trop. S. Paulo, 6:258-67, 1964.

18. TODD, E.C.D. Foodborne and waterborne disease in Canadá - 1979, Annual Summary. J. Food Protec., 48:1071-8, 1985.

19. WILSON, D. Pesquisa de Staphylococcus aureus em leite a ser pasteurizado. Rev. Saúde públ., S. Paulo, 11:1-11, 1977.

Recebido para publicação em 28/12/1987 Reapresentado em 4/8/1988

Aprovado para publicação em 9/8/1988 